\title{
Laplace-Type Semi-Invariants for a System of Two Linear Hyperbolic Equations by Complex Methods
}

\author{
F. M. Mahomed, ${ }^{1}$ A. Qadir, ${ }^{2}$ and A. Ramnarain ${ }^{1}$ \\ ${ }^{1}$ Centre for Differential Equations, Continuum Mechanics and Applications, School of Computational and \\ Applied Mathematics, University of the Witwatersrand, Wits 2050, South Africa \\ ${ }^{2}$ Centre for Advanced Mathematics and Physics, National University of Sciences and Technology, \\ Campus H-12, Islamabad 44000, Pakistan
}

Correspondence should be addressed to A. Qadir, aqadirmath@yahoo.com

Received 15 July 2011; Revised 21 September 2011; Accepted 26 September 2011

Academic Editor: F. Lobo Pereira

Copyright ( 2011 F. M. Mahomed et al. This is an open access article distributed under the Creative Commons Attribution License, which permits unrestricted use, distribution, and reproduction in any medium, provided the original work is properly cited.

In 1773 Laplace obtained two fundamental semi-invariants, called Laplace invariants, for scalar linear hyperbolic partial differential equations (PDEs) in two independent variables. He utilized this in his integration theory for such equations. Recently, Tsaousi and Sophocleous studied semiinvariants for systems of two linear hyperbolic PDEs in two independent variables. Separately, by splitting a complex scalar ordinary differential equation (ODE) into its real and imaginary parts PDEs for two functions of two variables were obtained and their symmetry structure studied. In this work we revisit semi-invariants under equivalence transformations of the dependent variables for systems of two linear hyperbolic PDEs in two independent variables when such systems correspond to scalar complex linear hyperbolic equations in two independent variables, using the above-mentioned splitting procedure. The semi-invariants under linear changes of the dependent variables deduced for this class of hyperbolic linear systems correspond to the complex semi-invariants of the complex scalar linear $(1+1)$ hyperbolic equation. We show that the adjoint factorization corresponds precisely to the complex splitting. We also study the reductions and the inverse problem when such systems of two linear hyperbolic PDEs arise from a linear complex hyperbolic PDE. Examples are given to show the application of this approach.

\section{Introduction}

In the study of scalar linear second-order partial differential equations (PDEs) in two independent variables, $x$ and $y$,

$$
A(x, y) u_{x x}+2 B(x, y) u_{x y}+C(x, y) u_{y y}+D(x, y) u_{x}+E(x, y) u_{y}+F(x, y) u=G(x, y)
$$


where $A$ to $G$ are $C^{2}$ functions in some domain and $u_{x}=\partial u / \partial x$ and so forth, it is well known that there are three canonical forms, namely, hyperbolic, parabolic, and elliptic (see, e.g. [1]), according to the sign of the discriminant $\Delta=B^{2}-A C$. Here we focus on the hyperbolic canonical form, which can be written as

$$
u_{x y}+a(x, y) u_{x}+b(x, y) u_{y}+c(x, y) u=0
$$

with $a, b$, and $c$ smooth functions in some region of the $x, y$ space.

One of the earliest studies of (1.2) is contained in Laplace's [2] memoir. Laplace deduced two fundamental quantities, called semi-invariants, for it:

$$
\begin{aligned}
& h=a_{x}+a b-c, \\
& k=b_{y}+a b-c,
\end{aligned}
$$

which were used for an integration theory of these equations. They remain invariant under point-wise scaling transformations of the dependent variable

$$
u=\sigma(x, y) \bar{u}, \quad \sigma \neq 0,
$$

but not under general transformations. They are also referred to in the literature as "Laplace invariants". They have also been used by Ovsiannikov [3] in the group classification of (1.2), where the determining equations for the symmetries of (1.2) were written in terms of $h$ and $k$.

The equivalence problem for scalar linear $(1+1)$ hyperbolic PDEs was solved separately in $[4,5]$. These works gave rise to further invariants apart from the Ovsiannikov invariants. Tsaousi and Sophocleous [6] obtained the analogue of the Laplace invariants for systems of two linear hyperbolic PDEs in two independent variables. They used the infinitesimal method, and it was shown that there are four differential invariants and five semi-invariants of order one (under changes of the dependent variables).

A method was developed for studying systems of two PDEs or ordinary differential equations (ODEs) by considering a complex scalar ODE and splitting it into its real and imaginary parts $[7,8]$. This was called "complex symmetry analysis" (CSA). These systems have fewer arbitrary coefficients than their classical analogues. The reduction is obtained because not all pairs of PDEs can be written as scalar complex PDEs since the coefficients satisfy the Cauchy-Riemann PDEs. It has been shown that such systems have operators that are inequivalent to those determined by the classical Lie approach [9].

In this work we revisit the study of semi-invariants of systems of two linear hyperbolic in two independent variables under dependent variable transformations using CSA. These are subclasses of systems of the form considered by Tsaousi and Sophocleous [6]. The motivation for this study is many fold. Firstly we deduce semi-invariants under linear changes of the dependent variables which correspond to the complex semi-invariants of the complex scalar linear $(1+1)$ hyperbolic PDE. These are shown to be special systems that arise from the scalar linear $(1+1)$ hyperbolic equations. We give precise conditions (see the Corollary) in terms of the coefficients and semi-invariants when the general system is reducible to scalar complex linear $(1+1)$ hyperbolic equations. The semi-invariants now are four and the eight $K_{i} \mathrm{~s}$ of [6] reduce to four semi-invariants $h_{1}, h_{2}, k_{1}$, and $k_{2}$. This solves 
the inverse problem of when such systems arise from the base complex scalar linear $(1+1)$ hyperbolic equations. Secondly we demonstrate that the special linear hyperbolic system which arises from a complex splitting of the scalar equation is factorizable if and only if its adjoint system is factorizable. We explicitly prove that this does not occur for the general system investigated in [6]. These results are contained in Theorems 3.4 and 3.5. New insights on factorization and reductions (Theorems 3.2 and 3.3) are obtained from here. We compare the semi-invariants obtained here with those of [6], including some examples. Thirdly the construction of the mappings that relate the two systems of hyperbolic PDEs of the class considered is easily done using the explicit dependent variable change for the scalar complex case which is in terms of the coefficients of the original and target PDEs (see Theorem 3.1). This is not the case for the general system studied in [6] as these explicit formulas are not as yet known for the general case.

The outline of this work is as follows. In the next section we look at equivalence transformations under dependent variables that relate two scalar linear $(1+1)$ hyperbolic PDEs and the special linear hyperbolic systems as a consequence. In Section 3 we study semiinvariants for our special system that arise by analytic continuation. Herein we also mention results on the factorization of the system and its adjoint as well as uncoupling. Mention is made of the inverse problem and when general hyperbolic systems arise from scalar hyperbolic PDEs. Our results are compared to those of [6]. Section 4 deals with examples that illustrate our approach. Finally we present a discussion of our results.

\section{Equivalence Transformations under Dependent Variables}

Equivalence transformations of a family of PDEs with arbitrary elements map the family into itself. We consider equivalence transformations of (1.2) under (1.4), which yield

$$
\bar{u}_{x y}+\bar{a}(x, y) \bar{u}_{x}+\bar{b}(x, y) \bar{u}_{y}+\bar{c}(x, y) \bar{u}=0
$$

where

$$
\begin{aligned}
& \bar{a}=a+\frac{\sigma_{y}}{\sigma}, \\
& \bar{b}=b+\frac{\sigma_{x}}{\sigma}, \\
& \bar{c}=\frac{L(\sigma)}{\sigma},
\end{aligned}
$$

$L$ being the linear operator

$$
L=\frac{\partial^{2}}{\partial x \partial y}+a \frac{\partial}{\partial x}+b \frac{\partial}{\partial y}+c
$$

so that (1.2) can be written compactly as $L u=0$. 
Now consider the linear hyperbolic system

$$
\begin{gathered}
v_{x y}=a_{1} v_{x}+b_{1} w_{x}+c_{1} v_{y}+d_{1} w_{y}+f_{1} v+g_{1} w, \\
w_{x y}=a_{2} v_{x}+b_{2} w_{x}+c_{2} v_{y}+d_{2} w_{y}+f_{2} v+g_{2} w,
\end{gathered}
$$

where $a_{i}$ to $g_{i}$ are arbitrary continuous functions of $x$ and $y$. Such systems were considered in [6]. It was shown there that the equivalence transformations of the dependent variables for system (2.4) are

$$
\begin{aligned}
v & =\rho_{1}(x, y) \bar{v}+\rho_{2}(x, y) \bar{w}, \\
w & =\rho_{3}(x, y) \bar{v}+\rho_{4}(x, y) \bar{w},
\end{aligned}
$$

in which $\rho_{i}$ are functions of $x$ and $y$. The new coefficients can be written in terms of the $\rho$ s and the old coefficients.

For the complex function

$$
u(x, y)=v(x, y)+i w(x, y)
$$

the ODE

$$
u^{\prime \prime}+A(z) u^{\prime}+B(z) u=0
$$

where $z=x+i y$, becomes a system of two linear second-order PDEs for $v$ and $w$ involving four arbitrary functions of $x$ and $y$ [7]. This is obviously a subclass of (2.4). Here we study a bigger subclass of (2.4), which comes from the complex split of (1.2). Thus we arrive at the special linear hyperbolic system

$$
\begin{aligned}
& L_{1} v-L_{2} w \equiv v_{x y}+a_{1} v_{x}-a_{2} w_{x}+b_{1} v_{y}-b_{2} w_{y}+c_{1} v-c_{2} w=0 \\
& L_{1} w+L_{2} v \equiv w_{x y}+a_{1} w_{x}+a_{2} v_{x}+b_{1} w_{y}+b_{2} v_{y}+c_{1} w+c_{2} v=0
\end{aligned}
$$

corresponding to a special class of equations obtainable by CSA for the complex operator

$$
L=L_{1}+i L_{2}
$$

in (2.3) with

$$
\begin{aligned}
& L_{1}=\frac{\partial^{2}}{\partial x \partial y}+a_{1} \frac{\partial}{\partial x}+b_{1} \frac{\partial}{\partial y}+c_{1} \\
& L_{2}=\frac{\partial^{2}}{\partial x \partial y}+a_{2} \frac{\partial}{\partial x}+b_{2} \frac{\partial}{\partial y}+c_{2}
\end{aligned}
$$


The subclass of equivalence transformations of the dependent variables (2.5) is derived by using the complex scaling function

$$
\sigma(x, y)=\sigma_{1}(x, y)+i \sigma_{2}(x, y)
$$

in (1.4) to arrive at

$$
\begin{array}{r}
v=\sigma_{1} \bar{v}-\sigma_{2} \bar{w}, \\
w=\sigma_{2} \bar{v}+\sigma_{1} \bar{w} .
\end{array}
$$

That this is a subclass of the more general transformations (2.5) is clear as there are only two arbitrary functions $\sigma_{1}$ and $\sigma_{2}$, whereas (2.5) has four arbitrary functions $\rho_{i}$. This subclass (2.12) transforms the system of linear PDEs (2.8) into

$$
\begin{aligned}
& \bar{L}_{1} \bar{v}-\bar{L}_{2} \bar{w}=0, \\
& \bar{L}_{1} \bar{w}+\bar{L}_{2} \bar{v}=0,
\end{aligned}
$$

in which $\bar{L}_{1}$ and $\bar{L}_{2}$ are as in (2.10) with the $a_{i}$ to $c_{i}$ in new coordinates (and thus with bars over them). Thus the linear transformations (2.12) are equivalence transformations of (2.8) provided

$$
\begin{aligned}
& \bar{a}_{1}=a_{1}+\frac{\sigma_{1} \sigma_{1 y}+\sigma_{2} \sigma_{2 y}}{\sigma_{1}^{2}+\sigma_{2}^{2}}, \\
& \bar{a}_{2}=a_{2}+\frac{\sigma_{1} \sigma_{2 y}-\sigma_{2} \sigma_{1 y}}{\sigma_{1}^{2}+\sigma_{2}^{2}}, \\
& \bar{b}_{1}=b_{1}+\frac{\sigma_{1} \sigma_{1 x}+\sigma_{2} \sigma_{2 x}}{\sigma_{1}^{2}+\sigma_{2}^{2}}, \\
& \bar{b}_{2}=b_{2}+\frac{\sigma_{1} \sigma_{2 x}-\sigma_{2} \sigma_{1 x}}{\sigma_{1}^{2}+\sigma_{2}^{2}}, \\
& \bar{c}_{1}=\frac{\sigma_{1}\left(L_{1} \sigma_{1}-L_{2} \sigma_{2}\right)+\sigma_{2}\left(L_{1} \sigma_{2}+L_{2} \sigma_{1}\right)}{\sigma_{1}^{2}+\sigma_{2}^{2}} \\
& \bar{c}_{2}=\frac{\sigma_{1}\left(L_{1} \sigma_{2}+L_{2} \sigma_{1}\right)-\sigma_{2}\left(L_{1} \sigma_{1}-L_{2} \sigma_{2}\right)}{\sigma_{1}^{2}+\sigma_{2}^{2}}
\end{aligned}
$$

The hyperbolic system (2.8) is a subclass of the system (2.4) considered in [6]. In (2.8) there are six arbitrary coefficients whereas in system (2.4) there are twelve. The special system (2.8) is uncoupled if the coefficients $a_{2}$ to $c_{2}$ are zero. One has

$$
\begin{gathered}
v_{x y}+a_{1} v_{x}+b_{1} v_{y}+c_{1} v=0 \\
w_{x y}+a_{1} w_{x}+b_{1} w_{y}+c_{1} w=0
\end{gathered}
$$


while the special coupled system is

$$
\begin{gathered}
v_{x y}-a_{1} w_{x}-b_{2} w_{y}-c_{2} w=0, \\
w_{x y}-a_{2} v_{x}+b_{2} v_{y}-c_{2} v=0 .
\end{gathered}
$$

We return to (2.15) in the next section.

In the following section we study the semi-invariants under the dependent variables changes (2.12) for the system (2.8). Furthermore we prove interesting properties on uncoupling, factorization, adjoint factorization, and the inverse problem.

\section{Semi-Invariants, Factorization, Adjoint Equations}

Laplace [2] stated the following theorem involving his semi-invariants (1.3).

\section{Laplace's Theorem}

The scalar linear hyperbolic PDE (1.2) is equivalent via (1.4) to the transformed hyperbolic PDE (2.1) if and only if

$$
h=\bar{h}, \quad k=\bar{k}
$$

where $\bar{h}$ and $\bar{k}$ are given by

$$
\begin{aligned}
& \bar{h}=\bar{a}_{x}+\bar{a} \bar{b}-\bar{c}, \\
& \bar{k}=\bar{b}_{y}+\bar{a} \bar{b}-\bar{c} .
\end{aligned}
$$

The construction of $\sigma$ in (1.4) is via the equations

$$
\begin{aligned}
& \bar{a}-a=\frac{\sigma_{y}}{\sigma}, \\
& \bar{b}-b=\frac{\sigma_{x}}{\sigma} .
\end{aligned}
$$

Clearly, one needs to know the coefficients of the target PDE (2.1) apart from the coefficients of the given equation. Note that the compatibility of this system (3.3) gives rise to a Laplace invariant.

We now determine the semi-invariants of the special linear hyperbolic system (2.8) which are invariant under the linear changes (2.12). Since it is obtained from the scalar 
hyperbolic PDE (1.2) considered as a complex PDE by the complex transformation (2.6), we can thus deduce the semi-invariants of (2.8) under (2.12) by setting

$$
\begin{aligned}
& h=h_{1}+i h_{2}, \\
& \bar{h}=\bar{h}_{1}+i \bar{h}_{2}, \\
& k=k_{1}+i k_{2}, \\
& \bar{k}=\bar{k}_{1}+i \bar{k}_{2} .
\end{aligned}
$$

The insertion of (3.4) into (1.3), (3.1), and (3.2) results in

$$
\begin{aligned}
& \bar{h}_{1}=h_{1}, \\
& \bar{h}_{2}=h_{2}, \\
& \bar{k}_{1}=k_{1}, \\
& \bar{k}_{2}=k_{2},
\end{aligned}
$$

where

$$
\begin{aligned}
& h_{1}=a_{1 x}+a_{1} b_{1}-a_{2} b_{2}-c_{1}, \\
& h_{2}=a_{2 x}+a_{1} b_{2}+a_{2} b_{1}-c_{2}, \\
& k_{1}=b_{1 y}+a_{1} b_{1}-a_{2} b_{2}-c_{1}, \\
& k_{2}=b_{2 y}+a_{1} b_{2}+a_{2} b_{1}-c_{2},
\end{aligned}
$$

and converts (3.6) to barred coordinates.

We can thus state the following theorem.

Theorem 3.1. The linear system of hyperbolic PDEs (2.8):

$$
\begin{aligned}
& L_{1} v-L_{2} w=0, \\
& L_{1} w+L_{2} v=0,
\end{aligned}
$$

is equivalent via (2.12)

$$
\begin{aligned}
v & =\sigma_{1} \bar{v}-\sigma_{2} \bar{w}, \\
w & =\sigma_{2} \bar{v}+\sigma_{1} \bar{w}
\end{aligned}
$$


to the transformed PDE system in barred coordinates (2.13) if and only if

$$
\begin{array}{ll}
\bar{h}_{1}=h_{1}, & \bar{h}_{2}=h_{2}, \\
\bar{k}_{1}=k_{1}, & \bar{k}_{2}=k_{2},
\end{array}
$$

where

$$
\begin{aligned}
& \bar{h}_{1}=\bar{a}_{1 x}+\bar{a}_{1} \bar{b}_{1}-\bar{a}_{2} \bar{b}_{2}-\bar{c}_{1}, \\
& \bar{h}_{2}=\bar{a}_{2 x}+\bar{a}_{1} \bar{b}_{2}+\bar{a}_{2} \bar{b}_{1}-\bar{c}_{2}, \\
& \bar{k}_{1}=\bar{b}_{1 y}+\bar{a}_{1} \bar{b}_{1}-\bar{a}_{2} \bar{b}_{2}-\bar{c}_{1}, \\
& \bar{k}_{2}=\bar{b}_{2 y}+\bar{a}_{1} \bar{b}_{2}+\bar{a}_{2} \bar{b}_{1}-\bar{c}_{2} .
\end{aligned}
$$

The $\sigma_{1}$ and $\sigma_{2}$ in (3.8) can be obtained from

$$
\begin{aligned}
& \bar{a}_{1}-a_{1}=\frac{\sigma_{1} \sigma_{1 y}+\sigma_{2} \sigma_{2 y}}{\sigma_{1}^{2}+\sigma_{2}^{2}}, \\
& \bar{a}_{2}-a_{2}=\frac{\sigma_{1} \sigma_{2 y}-\sigma_{2} \sigma_{1 y}}{\sigma_{1}^{2}+\sigma_{2}^{2}}, \\
& \bar{b}_{1}-b_{1}=\frac{\sigma_{1} \sigma_{1 x}+\sigma_{2} \sigma_{2 x}}{\sigma_{1}^{2}+\sigma_{2}^{2}}, \\
& \bar{b}_{2}-b_{2}=\frac{\sigma_{1} \sigma_{2 x}-\sigma_{2} \sigma_{1 x}}{\sigma_{1}^{2}+\sigma_{2}^{2}} .
\end{aligned}
$$

The proof of this result follows easily from the preceding discussion.

We note that for uncoupled systems, $a_{2}$ to $c_{2}$ in (2.8) are zero. Thus we have the result. Theorem 3.2. The linear system of hyperbolic PDEs (3.7) is reducible via (3.8) to the uncoupled system

$$
\begin{array}{r}
\bar{v}_{x y}+\bar{a}_{1} \bar{v}_{x}+\bar{b}_{1} \bar{v}_{y}+\bar{c}_{1} \bar{v}=0, \\
\bar{w}_{x y}+\bar{a}_{1} \bar{w}_{x}+\bar{b}_{1} \bar{w}_{y}+\bar{c}_{1} \bar{w}=0
\end{array}
$$

if and only if

$$
\begin{array}{ll}
\bar{h}_{1}=h_{1}, & \bar{h}_{2}=h_{2}=0, \\
\bar{k}_{1}=k_{1}, & \bar{k}_{2}=k_{2}=0 .
\end{array}
$$

We next review the idea of factorization and its implications for our system. These occur for normal and adjoint factorization. 
It is known from the works of Laplace [2] that the scalar hyperbolic PDE (1.2) is factorizable in terms of first-order linear operators if and only if $h=0$ or $k=0$. That is, the PDE (1.2) can be written as

$$
\left(\frac{\partial}{\partial x}+b\right)\left(\frac{\partial}{\partial y}+a\right) u=0
$$

if $h=0$ and

$$
\left(\frac{\partial}{\partial y}+a\right)\left(\frac{\partial}{\partial x}+b\right) u=0
$$

if $k=0$. If both $h$ and $k$ are zero, then the factors commute and (1.2) in this case is reducible via dependent variable transform (1.4) to the simplest wave equation $\bar{u}_{x y}=0$. We translate these results to the special system of PDEs (3.7).

We therefore have the next theorem.

Theorem 3.3. The hyperbolic system (3.7), by analytic continuation, corresponds to the scalar factorizable PDE (3.14) or (3.15) if and only if $h_{1}=h_{2}=0$ or $k_{1}=k_{2}=0$. In the case $h_{1}=h_{2}=0$, the linear hyperbolic system

$$
\begin{gathered}
v_{x y}+\left(a_{1} v\right)_{x}-\left(a_{2} w\right)_{x}+b_{1} v_{y}+a_{1} b_{1} v-b_{1} a_{2} w-b_{2} w_{y}-a_{1} b_{2} w-a_{2} b_{2} v=0 \\
w_{x y}+\left(a_{1} w\right)_{x}+\left(a_{2} v\right)_{x}+b_{1} w_{y}+a_{1} b_{1} w+b_{1} a_{2} v+b_{2} v_{y}+a_{1} b_{2} v-a_{2} b_{2} w=0
\end{gathered}
$$

corresponds to (3.14). For $k_{1}=k_{2}=0$ the system

$$
\begin{gathered}
v_{x y}+\left(b_{1} v\right)_{y}-\left(b_{2} w\right)_{y}+a_{1} v_{x}+a_{1} b_{1} v-a_{1} b_{2} w-a_{2} w_{x}-a_{2} b_{1} w-a_{2} b_{2} w=0 \\
w_{x y}+\left(b_{1} w\right)_{y}+\left(b_{2} w\right)_{x}+a_{1} w_{x}+a_{1} b_{1} w+a_{1} b_{2} w+a_{2} v_{x}+b_{1} a_{2} v-a_{2} b_{2} w=0
\end{gathered}
$$

corresponds to (3.15).

We now state and prove a result that is apparently not known in the literature on adjoint equations which is important in the construction of the Riemann function. Riemann's method for $(1+1)$ linear hyperbolic PDEs utilizes the adjoint equation to arrive at the exact solution of an initial value problem (see, e.g [1]). In this approach the solution depends on the adjoint equation with specified boundary conditions being solved. Hence it of significance to have properties of adjoint hyperbolic equations. We thus have the following result.

Theorem 3.4. The hyperbolic PDE (1.1) is factorizable in terms of first-order linear operators if and only if its adjoint equation, namely,

$$
u_{x y}-a u_{x}-b u_{y}+\left(c-a_{x}-b_{y}\right) u=0,
$$

is factorizable. 
Proof. The proof follows by noting that if $h$ and $k$ are semi-invariants of (1.1) and if $h$ or $k$ is zero, then the adjoint equation (3.18) has Laplace invariants $h^{\prime}=k$ and $k^{\prime}=h$, one of which is zero. The converse similarly applies.

It should be mentioned that this property of adjoint factorization does not in general apply to $(1+1)$ linear parabolic equations

$$
u_{t}=a u_{x x}+b u_{x}+c u,
$$

where $a$ to $c$ are smooth functions. This PDE (3.19) has semi-invariants, under linear change of dependent variable, $a$ and $K$ (see $[10,11]$ ). The adjoint equation to (3.19) is

$$
u_{t}=-a u_{x x}+\left(b-2 a_{x}\right) u_{x}+\left(b_{x}-a_{x x}-c\right) u
$$

It is clear that the semi-invariant $a$ becomes $-a$. Also the expression for $K$ in (3.20) is more complicated and not the same as $K$. These can be more easily appreciated via some telling examples.

It is interesting that the adjoint factorization property when the parent equation is factorizable can be transferred to our special system. Indeed we have the following theorem.

Theorem 3.5. The special linear hyperbolic system (2.8) is factorizable if and only if its adjoint system, namely,

$$
\begin{gathered}
v_{x y}-a_{1} v_{x}+a_{2} w_{x}-b_{1} v_{y}+b_{2} w_{y}+\left(c_{1}-a_{1 x}-b_{1 y}\right) v-\left(c_{2}-a_{2 x}-b_{2 y}\right) w=0 \\
w_{x y}-a_{1} w_{x}-a_{2} v_{x}-b_{1} w_{y}-b_{2} v_{y}+\left(c_{1}-a_{1 x}-b_{1 y}\right) w+\left(c_{2}-a_{2 x}-b_{2 y}\right) v=0
\end{gathered}
$$

\section{is factorizable.}

Proof. The proof follows from noting that the adjoint condition is invariant under the complex split. For the analytic continuation of the adjoint scalar PDE (3.20) defined in the complex domain gives rise to (3.21) which is precisely the adjoint system of the special class (2.8). Thus the factorization property of Theorem 3.4 applies due to the complex split.

It is now opportune to recall the results of [6]. In this paper, inter alia, it was shown that the system (2.4) has the Laplace-type invariants

$$
\begin{aligned}
& I_{1}=K_{1}+K_{4}, \\
& I_{2}=K_{5}+K_{8}, \\
& I_{3}=K_{1} K_{4}-K_{2} K_{3}, \\
& I_{4}=K_{5} K_{8}-K_{6} K_{7}, \\
& I_{5}=K_{5} K_{1}+K_{2} K_{7}+K_{3} K_{6}+K_{4} K_{8},
\end{aligned}
$$


where

$$
\begin{aligned}
& K_{1}=a_{1} c_{1}+a_{2} d_{1}-a_{1 x}+f_{1}, \\
& K_{2}=a_{1} c_{2}+a_{2} d_{2}-a_{2 x}+f_{2}, \\
& K_{3}=b_{1} c_{1}+b_{2} d_{1}-b_{1 x}+g_{1}, \\
& K_{4}=b_{1} c_{2}+b_{2} d_{2}-b_{2 x}+g_{2}, \\
& K_{5}=a_{1} c_{1}+b_{1} c_{2}-c_{1 y}+f_{1}, \\
& K_{6}=a_{2} c_{1}+b_{2} c_{2}-c_{2 y}+f_{2}, \\
& K_{7}=a_{1} d_{1}+b_{1} d_{2}-d_{1 y}+g_{1}, \\
& K_{8}=a_{2} d_{1}+b_{2} d_{2}-d_{2 y}+g_{2} .
\end{aligned}
$$

It is not difficult to see that for our system (2.8), $K_{1}$ to $K_{8}$ reduce to just the four semiinvariants we obtained above, namely, $h_{1}$ to $k_{2}$. Hence the semi-invariants (3.22) can be written in terms of just four quantities $h_{1}$ to $k_{2}$ as

$$
\begin{aligned}
& I_{1}=2 h_{1}, \\
& I_{2}=2 k_{1}, \\
& I_{3}=h_{1}^{2}+h_{2}^{2}, \\
& I_{4}=k_{1}^{2}+k_{2}^{2}, \\
& I_{5}=2 h_{1} k_{1}-2 h_{2} k_{2} .
\end{aligned}
$$

A similar theorem to Theorem 3.5 does not apply to the more general system (2.4) considered in [6]. This can be seen as follows. The adjoint system to (2.4) is

$$
\begin{aligned}
& v_{x y}=-a_{1} v_{x}-b_{1} w_{x}-c_{1} v_{y}-d_{1} w_{y}-\left(a_{1 x}+c_{1 y}-f_{1}\right) v-\left(b_{1 x}+d_{1 y}-g_{1}\right) w \\
& w_{x y}=-a_{2} v_{x}-b_{2} w_{x}-c_{2} v_{y}-d_{2} w_{y}-\left(a_{2 x}+c_{2 y}-f_{2}\right) v-\left(b_{2 x}+d_{2 y}-g_{2}\right) w
\end{aligned}
$$

This system has the values of $K^{\prime}$ s given by

$$
\begin{aligned}
& K_{1}=a_{1} c_{1}+a_{2} d_{1}-c_{1 y}+f_{1}, \\
& K_{2}=a_{1} c_{2}+a_{2} d_{2}-c_{2 y}+f_{2}, \\
& K_{3}=b_{1} c_{1}+b_{2} d_{1}-d_{1 y}+g_{1}, \\
& K_{4}=b_{1} c_{2}+b_{2} d_{2}-d_{2 y}+g_{2},
\end{aligned}
$$




$$
\begin{aligned}
& K_{5}=a_{1} c_{1}+b_{1} c_{2}-a_{1 x}+f_{1}, \\
& K_{6}=a_{2} c_{1}+b_{2} c_{2}-a_{2 x}+f_{2}, \\
& K_{7}=a_{1} d_{1}+b_{1} d_{2}-b_{1 x}+g_{1}, \\
& K_{8}=a_{2} d_{1}+b_{2} d_{2}-b_{2 x}+g_{2} .
\end{aligned}
$$

Only $I_{1}$ and $I_{2}$ remain the same for the adjoint system (3.25). They just become interchanged. The semi-invariants $I_{3}$ to $I_{5}$ are in general not preserved (the extreme case in which they are the same is when the system is self-adjoint which occurs if and only if the $f \mathrm{~s}$ and $g \mathrm{~s}$ are nonzero with the remaining coefficients zero). So indeed Theorem 3.5 is a special property only enjoyed by systems that arise from the complex split of a scalar linear hyperbolic PDE.

Corollary 3.6. The general system (2.4) arises from the complex continuation of the scalar linear $(1+1)$ hyperbolic PDE (1.1) if and only if its coefficients are precisely of the form (2.8) or equivalently if (2.4) has quantities $K_{i} s$ which are written solely in terms of the semi-invariants $h_{1}, h_{2}, k_{1}$, and $k_{2}$ as

$$
\begin{array}{cl}
K_{1}=h_{1}, \quad K_{2}=h_{2}, & K_{3}=-h_{2}, \quad K_{4}=h_{1}, \quad K_{5}=k_{1}, \quad K_{6}=k_{2}, \\
K_{7}=-k_{2}, & K_{8}=k_{1} .
\end{array}
$$

This Corollary also solves the inverse problem of when systems of the form (2.4) arise from a scalar linear $(1+1)$ hyperbolic PDE defined in the complex plane.

\section{Illustrative Examples}

We present a few illustrative examples some of which are taken from [6] for comparison.

(1) The uncoupled system is

$$
\begin{aligned}
v_{x y}+(x v)_{x}+y v_{y}+x y v & =0 \\
w_{x y}+(x w)_{x}+y w_{y}+x y w & =0
\end{aligned}
$$

which has $h_{1}=h_{2}=0=k_{1}=k_{2}$ and is reducible to the simplest system $\bar{v}_{x y}=0=\bar{w}_{x y}=0$ by means of the linear transformation

$$
\begin{aligned}
v & =\bar{v} \exp (-x y) \\
w & =\bar{w} \exp (-x y)
\end{aligned}
$$

Note that Theorem 1 of [6] also applies here. However, this system arises from a complex hyperbolic PDE. 
(2) Consider now the coupled system

$$
\begin{array}{r}
v_{x y}+(x v)_{x}+y v_{y}+x y v-x w w_{y}-x^{2} w=0 \\
w_{x y}+(x w)_{x}+y w_{y}+x y w+x v_{y}+x^{2} v=0 .
\end{array}
$$

This system has semi-invariants $h_{1}=h_{2}=0=k_{1}=k_{2}$ and hence can be again transformed to the simplest system. the transformation that does the reduction is

$$
\begin{aligned}
& v=\exp (-x y)\left(\frac{\bar{v} \cos x^{2}}{2}+\frac{\bar{w} \sin x^{2}}{2}\right) \\
& w=\exp (-x y)\left(-\frac{\bar{v} \sin x^{2}}{2}+\frac{\bar{w} \cos x^{2}}{2}\right)
\end{aligned}
$$

A similar comment as in example (1) can be made here too.

(3) The system of linear hyperbolic PDEs

$$
\begin{array}{r}
v_{x y}+x v_{x}-y w_{x}+y v_{y}-x w_{y}+x y v=0 \\
w_{x y}+x w_{x}+y v_{x}+y w_{y}+x v_{y}+x y w=0
\end{array}
$$

can be reduced via

$$
\begin{aligned}
& v=\exp (-x y)\left[\bar{v} \cos \left(\frac{x^{2}}{2}+\frac{y^{2}}{2}\right)+\bar{w} \sin \left(\frac{x^{2}}{2}+\frac{y^{2}}{2}\right)\right] \\
& w=\exp (-x y)\left[-\bar{v} \sin \left(\frac{x^{2}}{2}+y^{2}\right)+\bar{w} \cos \left(\frac{x^{2}}{2}+\frac{y^{2}}{2}\right)\right]
\end{aligned}
$$

to the simpler system

$$
\begin{gathered}
\bar{v}_{x y}+(x y-1) \bar{v}+\left(x^{2}+y^{2}\right) \bar{w}=0, \\
\bar{w}_{x y}+(x y-1) \bar{w}-\left(x^{2}+y^{2}\right) w=0,
\end{gathered}
$$

since $h_{1}=1-x y=k_{1}$ and $h_{2}=x^{2}+y^{2}=k_{2}$.

(4) The system

$$
\begin{array}{r}
v_{x y}+(x v)_{x}-(y w)_{x}+x v_{y}+x^{2} v+y w_{y}+y^{2} v=0 \\
w_{x y}+(x w)_{x}+(y v)_{x}+x w_{y}+x^{2} w-y v_{y}+y^{2} w=0
\end{array}
$$

has semi-invariants $h_{1}=0=h_{2}$ and $k_{1}=-1=k_{2}$. Therefore it is factorizable as

$$
\left(\frac{\partial}{\partial x}+x-i y\right)\left(\frac{\partial}{\partial y}+x+i y\right) u=0
$$


This last PDE can be treated as a system of two linear first-order PDEs for its solution. Here we have $I_{1}=I_{3}=I_{5}=0$ so that Theorem 4 of [6] is satisfied too. Thus this system can be factorizable in two ways. One is via the scalar complex factored PDE (4.9) and the other as factorization of each of the two equations comprising the system as in Theorem 4 of [6].

(5) The system

$$
\begin{array}{r}
2 v_{x y}+(x+1) v_{x}-(x-1) w_{x}+2 y v_{x}+(y x+y+1) v-(y x-y+1) w=0 \\
2 w_{x y}-(x-1) v_{x}+(x+1) w_{x}+2 y w_{y}-(y x-y+1) v+(y x+y+1) w=0
\end{array}
$$

was considered in [6] for factorization. However, it is not of the form (2.8) (the Corollary does not apply here), and hence it cannot be reducible to a complex scalar hyperbolic PDE.

\section{Discussion}

In this work we have used complex splitting of the scalar linear $(1+1)$ hyperbolic equation to transform it into a system of two linear hyperbolic equations by a complex split. The equivalence transformations of the dependent variable that maps the scalar complex linear $(1+1)$ hyperbolic PDE to itself also transform the system that arises from the complex scalar PDE to itself. Usually the algebraic properties do not transfer to systems by complex splitting [9]. The Laplace-type invariants were then found for this special system. These four Laplacetype invariants arise from the two Laplace invariants of the scalar linear $(1+1)$ hyperbolic PDE. We then focused on reductions to simpler systems using these semi-invariants (see Theorems 3.2 and 3.3). In particular we considered uncoupling and factorization of systems for which we obtained new results in the sense that they relate to scalar base equations.

We found that our special system has adjoint equations which are factorizable in terms of the scalar PDE from which it arises if the parent equation is factorizable as well (see Theorems 3.4 and 3.5). It was shown that this property of adjoint factorization does not hold for more general systems as in [6] but precisely those that arise from the complex linear hyperbolic $P D E$. This is not surprising as a self-adjoint operator is Hermitian and that is what is required for the real system to correspond to a complex scalar base equation.

We also pointed out how our system fits into the more general system considered in [6]. As a consequence we have provided the answer to the inverse problem of when linear hyperbolic systems of two equations in two independent variables arise from a scalar complex linear $(1+1)$ hyperbolic PDE as given in the Corollary. Moreover the transformation that relates the special systems are easily constructible (see Theorem 3.1) by using the base transformations which are in terms of the coefficients of the original and target PDEs. This is not as yet known for general systems as in [6]. Many examples were given to illustrate our method. Some of these were also related to the examples given in [6].

\section{Acknowledgments}

F. M. Mahomed thanks NUST CAMP for providing an enabling environment and hospitality during which time this work was done. He is also grateful to the HEC of Pakistan for a visiting professorship. A. Qadir is grateful to DECMA and CAM of Wits for support during a visit. 


\section{References}

[1] I. N. Sneddon, Elements of Partial Differential Equations, Dover, NY, USA, 1957.

[2] P. S. Laplace, Recherches sur le Calcul Intégral aux Différences Partielles, Mémoires de l'Académie Royale des Sciences de Paris, 1773.

[3] L. V. Ovsiannikov, Group Analysis of Differential Equations, Academic Press, New York, NY, USA, 1982.

[4] N. H. Ibragimov, "Invariants of hyperbolic equations: a solution of the Laplace problem," Journal of Applied Mechanics and Technical Physics, vol. 45, pp. 158-166, 2004.

[5] I. K. Johnpillai and F. M. Mahomed, "Equivalence problem for scalar linear (1+1) hyperbolic equations," Problems of Nonlinear Analysis in Engineering Systems, vol. 11, no. 22, pp. 63-74, 2005.

[6] C. Tsaousi and C. Sophocleous, "Differential invariants for systems of linear hyperbolic equations," Journal of Mathematical Analysis and Applications, vol. 363, no. 1, pp. 238-248, 2010.

[7] S. Ali, F. M. Mahomed, and A. Qadir, "Linearizability criteria for systems of two second-order differential equations by complex methods," Nonlinear Dynamics, vol. 66, no. 1-2, pp. 77-88, 2011.

[8] S. Ali, F. M. Mahomed, and A. Qadir, "Complex Lie symmetries for scalar second-order ordinary differential equations," Nonlinear Analysis: Real World Applications, vol. 10, no. 6, pp. 3335-3344, 2009.

[9] S. Ali, A. Qadir, and M. Safdar, "Inequivalence of classes of linearizable systems of cubically semi linear ordinary differential equations obtained by real and complex symmetry analysis," Mathematical and Computational Applications, vol. 16, pp. 923-934, 2011.

[10] N. H. Ibragimov, Elementary Lie Group Analysis and Ordinary Differential Equations, John Wiley and Sons, New York, NY, USA, 1999.

[11] N. H. Ibragimov, "Laplace type invariants for parabolic equations," Nonlinear Dynamics, vol. 28, no. 2, pp. 125-133, 2002. 


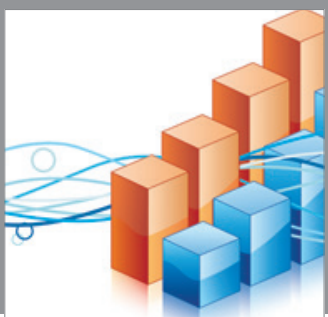

Advances in

Operations Research

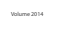



\section{The Scientific} World Journal
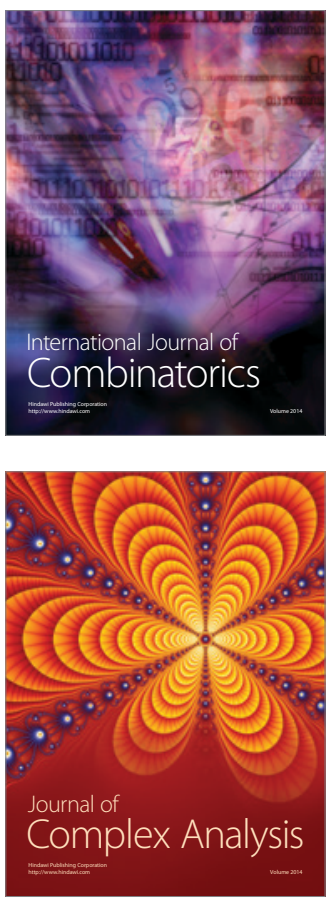

International Journal of

Mathematics and

Mathematical

Sciences
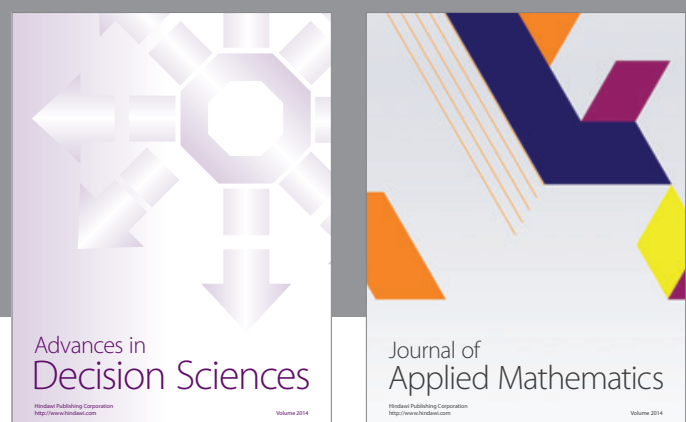

Journal of

Applied Mathematics


Submit your manuscripts at http://www.hindawi.com
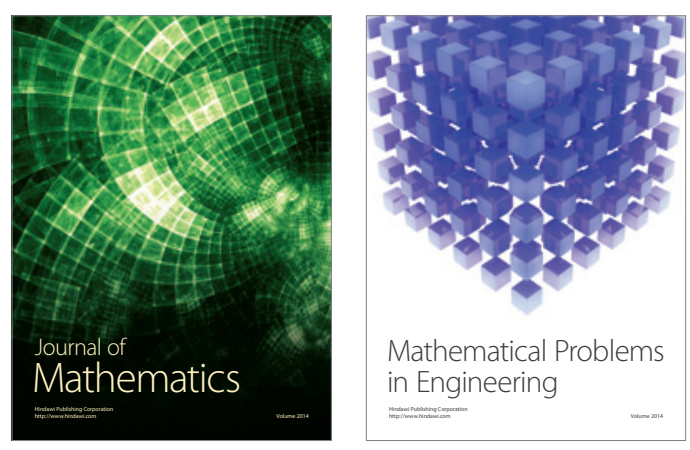

Mathematical Problems in Engineering
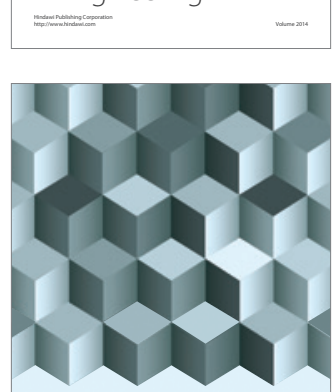

Journal of

Function Spaces


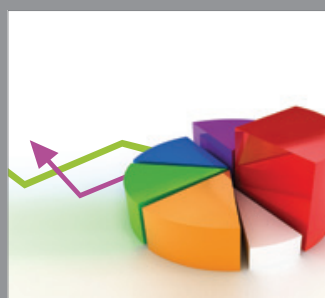

ournal of

Probability and Statistics

Promensencen
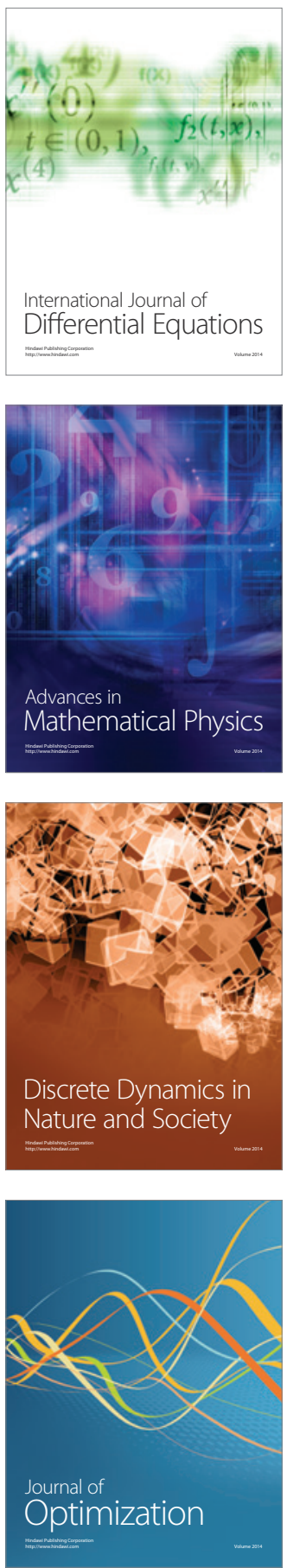